\title{
Instability of magnetic fluid in a narrow gap between plates
}

\author{
Viktor Polevikov $^{\mathrm{a}}$, Lutz Tobiska ${ }^{\mathrm{b}, *}$ \\ ${ }^{a}$ Belarussian State University, 4 F. Skaryna Avenue, 220027 Minsk, Belarus \\ b Otto-von-Guericke-Universität, Postfach 4120, D-39016 Magdeburg, Germany
}

Available online 7 December 2004

\begin{abstract}
The behaviour of a layer of magnetic fluid in a plane capillary subjected to a uniform magnetic field is studied numerically. Two different types of instabilities arise as the intensity of the magnetic field increases. For small contact angles the layer breaks along the capillary axis and spreads over the capillary wall whereas for large contact angles the layer elongates in the central part up to its separation from the walls. Critical parameters and shapes are studied by solving the coupled nonlinear system of differential equations numerically.
\end{abstract}

(C) 2004 Elsevier B.V. All rights reserved.

PACS: 47.55.Dz; 75.50.Mm; 47.20.Ma; 02.70.Bf; 02.70.Pt

Keywords: Magnetic fluids; Instability; Maxwell equations; Young-Laplace equation; Numerical simulation

\section{Introduction}

The influence of a vertical uniform magnetic field on the equilibrium state of a magnetic fluid drop in a capillary has been considered in Ref. [1]. By means of an ellipsoid approximation it has been shown theoretically that two types of instability arise as the intensity of the magnetic field increases. In the case of full wetting (contact angle equals zero) the drop breaks along the capillary axis and spreads over the capillary wall. If the fluid is non-wetting (contact angle equals $180^{\circ}$ ) the drop elongates in the central part up to its separation from the walls. The last type of instability has been also observed in experiments.

The main objective of the paper is to derive a model for this phenomenon in a wide range of contact angles

\footnotetext{
*Corresponding author. Ruhn Universitat Bochum, Universitatsstrasse 150, Bochum 44780, Germany. Tel.: + 493916718650 ; fax: +493916718073 .

E-mail address: tobiska@mathematik.uni-magdeburg.de (L. Tobiska).
}

on the basis of the balance equations in ferrohydrodynamics. Our model consists of the Young-Laplace equation which defines the shape of the free surface and the Maxwell equations which describe the structure of the magnetic fields in the fluid and the surrounding media. The deformation of the fluid layer in a narrow gap of thickness 2 a subjected to a uniform magnetic field perpendicular to the undisturbed layer will be studied. We suppose that the two free surfaces between two nonmagnetic parallel plates are two-dimensional, symmetric and bounded by a non-magnetic gas. We consider only plane perturbations of the magnetic field, thus the problem can be reduced to a $2 \mathrm{D}$ problem in the crosssection $\Omega$.

\section{Governing equations}

The Maxwell equations for a non-conducting fluid are given by

curl $H=0, \operatorname{div} B=0$, in $\Omega$ 
with the magnetic field strength $H$ and the magnetic induction $B$ satisfying the constitutive relation

$B=\mu_{0}(M+H)$ in $\Omega_{\mathrm{f}}$,

$B=\mu_{0} H$ outside $\Omega_{\mathrm{f}}$,

where $M$ is the magnetization, $\mu_{0}=4 \pi \times 10^{-2} \mathrm{Vs} / \mathrm{Am}$ is the permeability constant, $\Omega_{\mathrm{f}}$ is the domain occupied by the magnetic fluid. The magnetization is assumed to be parallel to the magnetic field and to follow the Langevin law [2-4]

$M=M_{\mathrm{S}}\left(\operatorname{coth}\left(\frac{|H|}{H^{*}}\right)-\frac{H^{*}}{|H|}\right) \frac{H}{|H|}$,

with the saturation magnetization $M_{\mathrm{S}}$, the Langevin parameter $H^{*}=M_{\mathrm{S}} /(3 \chi)$, and the initial susceptibility $\chi$. The Young-Laplace equation represents the force balance at the free surfaces and can be written in the form [5]

$$
\begin{aligned}
\sigma K= & \frac{\mu_{0}}{2}(M n)^{2} \\
& +\mu_{0} M_{\mathrm{S}} H^{*}\left(\ln \sinh \frac{|H|}{H^{*}}-\ln \frac{|H|}{H^{*}}\right)+\text { const },
\end{aligned}
$$

where $\sigma$ is the surface tension coefficient, $K$ the curvature, $n$ the surface normal, and const a constant which has to be fixed by the given area $\left|\Omega_{\mathrm{f}}\right|$ of the fluid cross section and the contact angle $\alpha$. The coupled nonlinear boundary value problem is defined by the five dimensionless parameters: $W=\mu_{0} M_{\mathrm{S}}^{2} a /(2 \sigma), U=$ $\left|\Omega_{\mathrm{f}}\right| / a^{2}, h_{\infty}=H_{0} / H^{*}, \chi$, and $\alpha$.

\section{Solution method}

The Maxwell equation can be solved by introducing the magnetic potential $\varphi$ with $H=\nabla \varphi$. In the exterior of $\Omega_{\mathrm{f}}$, the magnetic potential is the solution of a linear elliptic equation with given boundary condition at infinity. In the interior of $\Omega_{\mathrm{f}}$ a nonlinear elliptic equation for the magnetic potential has to be solved due to nonlinear material law. At the interface we require continuity of both the magnetic potential $\varphi$ and the normal component of $B$. The coupled problem for the magnetic potential is solved by a combination of the boundary element method [6] with the finite element method (BEM-FEM approach) which allows to satisfy the far field condition at infinity and to handle the nonlinear magnetization law within the fluid.

The solution of the magneto-static problem for a given position of the free surface is only one step in the exterior iteration. The second step consists in the calculation of a new free surface satisfying the YoungLaplace equation for a given right-hand side, i.e., in our $2 \mathrm{D}$ setting we look for a curve with given curvature. Let the parametrization of this curve be given by $x=$ $x(s), y=y(s), s \in[0, L]$ we end up with the following second order system of ordinary differential equations $[7,8]$

$x^{\prime \prime}+y^{\prime}(F+$ const $)=0$,

$y^{\prime \prime}-x^{\prime}(F+$ const $)=0,0 \leqslant s \leqslant L$,

which has to be completed by appropriate boundary conditions and the requirement of a fixed area of the cross section occupied by the fluid layer. This system is solved by a second order finite difference scheme on an adaptive grid. A similar approach has been applied successfully to study the normal field instability [9].

\section{Results of numerical simulations}

In the following we present the results for the parameter set $\chi=5, U=4$, and $W=100$. Both types of instabilities could be detected numerically. The switch from one type to the other has been observed at the contact angle $\alpha=\alpha^{*}=117^{\circ}$. The first type of instability (spreading on the walls) appears for $\alpha<\alpha^{*}$ the second type (separation from the walls) for $\alpha>\alpha^{*}$. Further numerical calculations indicate that the critical value for the contact angle $\alpha=\alpha^{*}$ does not depend on the parameter $\mathrm{W}$ and only weakly on the susceptibility $\chi$.

Fig. 1 presents the critical values of the magnetic intensity parameter $h_{\infty}^{*}$ at which instability occurs. The regions of instability correspond to the domains above the two curves. The expected configurations of the magnetic fluid layer in these domains are indicated. The contact angle $\alpha$ has a strong influence on the critical intensity of the magnetic field. In the ranges $\alpha<\alpha^{*}$ and $\alpha>\alpha^{*}$ the maximum of the critical parameter $h_{\infty}^{*}$ is achieved when $\alpha \rightarrow \alpha^{*}$ and the minimum at $\alpha=0^{\circ}$ and

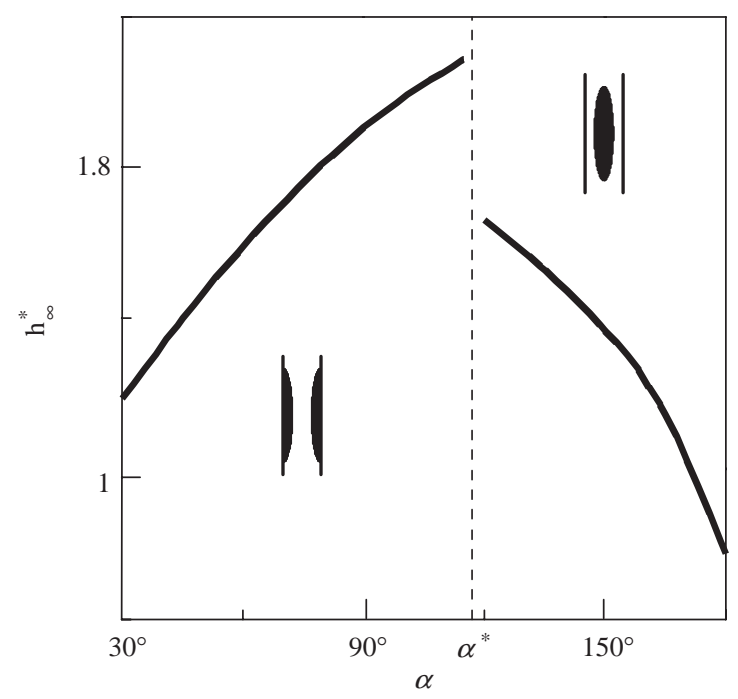

Fig. 1. Influence of the contact angle $\alpha$ on the critical value of the intensity parameter $h_{\infty}^{*}$ of the applied field. 


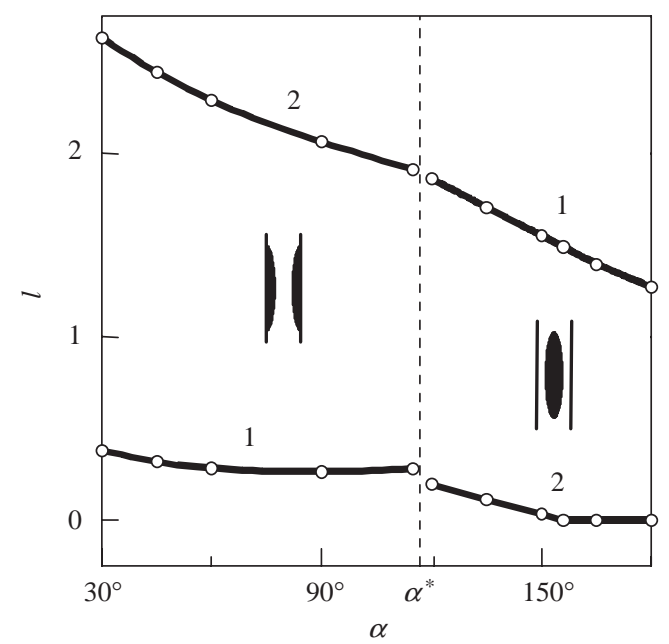

Fig. 2. Critical layer thickness as a function of the contact angle $\alpha$. For values $\alpha<\alpha^{*}$ the fluid layer breaks suddenly and spreads over the walls whereas for $\alpha<155^{\circ}$ the layer separates suddenly from the walls.
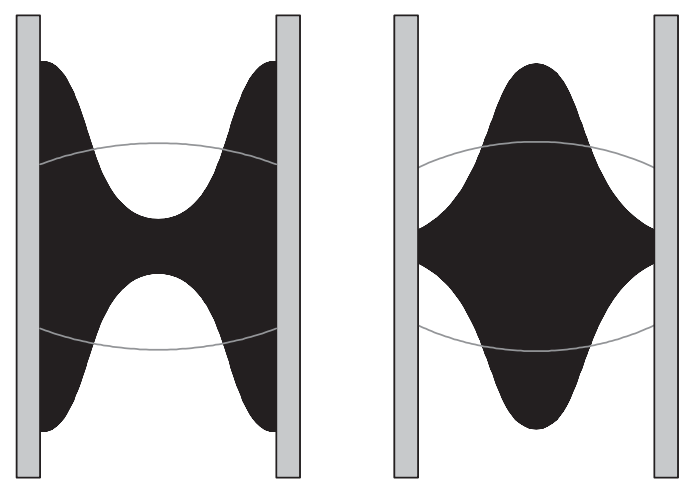

Fig. 3. Critical shapes of the free surface for two values of the contact angle, $\alpha=115^{\circ}$ (left) and $\alpha=120^{\circ}$ (right).

$\alpha=180^{\circ}$, respectively. In each of the ranges the maximum is about two times of the minimum value.

In Fig. 2 two values of the critical layer thickness are shown as a function of the contact angle $\alpha$ where $l$ denotes the ratio of the layer thickness to the gap width: 1 , in the central section; 2 , at the wall. Note that for $\alpha<\alpha^{*}$ the layer is more elongated than for $\alpha>\alpha^{*}$. The critical minimal thickness $l$ depends only weakly on the contact angle for $\alpha<\alpha^{*}$ and achieves values of $0.26-0.38$ in the interval $30^{\circ} \leqslant \alpha \leqslant 115^{\circ}$. In the range $\alpha^{*}<\alpha<155^{\circ}$ the critical minimal thickness tends to zero if the contact angle $\alpha$ increases. Thus, in the interval $155^{\circ}<\alpha \leqslant 180^{\circ}$ the fluid does not suddenly separate from the walls as it is the case for smaller contact angles.

Critical shapes of the magnetic fluid layer which correspond to the values $\alpha=115^{\circ}<\alpha^{*}$ (left) and $\alpha=$ $120^{\circ}>\alpha^{*}$ (right) for $h_{\infty}=h_{\infty}^{*}$ are illustrated in Fig. 3 .

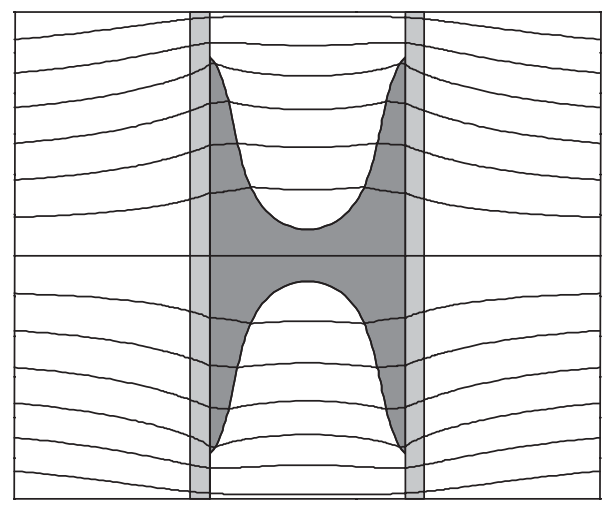

Fig. 4. Isolines of the dimensionless magnetic potential $\varphi$ in the range $-2.4 \leqslant \varphi \leqslant+2.4$.

The isolines of the dimensionless magnetic potential $\varphi$ for $\alpha=45^{\circ}$ and $h_{\infty}=h_{\infty}^{*}$ are shown in Fig. 4. Note that the magnetic field both inside and outside the fluid is non-uniform. Thus, the ellipsoid approximation seems to be not sufficient to model this case.

\section{Acknowledgement}

The authors like to thank the German Research Foundation (DFG-436 WER 17/8/03) for supporting this work.

\section{References}

[1] V. Bashtovoi, P. Kuzhir, A. Reks, J. Magn. Magn. Mater. 252 (2002) 265.

[2] B.M. Berkovski, V. Bashtovoi (Eds.), Magnetic Fluids and Application Hand Book, Begell House Inc. Publ., 1996.

[3] B.M. Berkovski, V.F. Medvedev, M.S. Krakov, Magnetic Fluids, Oxford University Press Inc., Oxford, 1993.

[4] E. Blum, A. Cebers, M.M. Maiorov, Magnetic Fluids, Walter de Gruyter, Berlin, 1997.

[5] L.E. Rosensweig, Ferrohydrodynamics, Dover Publ. Inc., New York, 1997.

[6] L.C. Wrobel, The boundary element method, Applications in Thermofluids and Acustics, Wiley, New York, 2002.

[7] V.K. Polevikov, Differential Equations 35 (1999) 985.

[8] V.K. Polevikov, Report 10-98, Otto-von-Guericke Univerisity of Magdeburg, 1998.

[9] V. Bashtovoi, O.A. Lavrova, V.K. Polevikov, L. Tobiska, J. Magn. Magn. Mater. 252 (2002) 299.

\section{Further reading}

[10] C.A. Brebbia, J.C.F. Telles, L.C. Wrobel, Boundary element techniques, Theory and Applications in Engineering, Springer, Berlin, 1984. 